722; Smith, 298; Rayleigh, 298; Mayhew, 567; Chauvenet, 40, 41; Danish Academy, 297; Franklin Institute, 403; Graustein, 567; Lobachevsky, 177; Mahamaya, 90; A. Cressy Morrison, 722; Naples Academy, 567; Nobel, 178; Paris Academy of Sciences, 177; Quebec Government, 178; Reale Accademia dei Lincei, 90; Royal Astronomical Society, 178; Royal Society of Edinburgh, 567; Royal Society of London, 178; Società Italiana delle Scienze, 177.

Universities and Technical Schools:

Cambridge, 90, 298, 567, 568, 722;

Chicago, 180 ;

Columbia, 181;

Minnesota, 182;

Cornell, 181;

Ohio State, 182;

Illinois, 181;

Oxford, 90, 700;

Iowa, 181;

Pennsylvania, 182;

Jerusalem, 723;

Princeton, 183;

Johns Hopkins, 181, 721, 723;

Rutgers, 298;

Stanford, 182;

Massachusetts Institute of Technology, 183;

Michigan, 181;

Texas, 182;

Tokyo, 566;

Wisconsin, 182.

\title{
ERRATA-VOLUME XXXII
}

F. S. Nowlan, Representation of integers by certain ternary cubic forms.

Page 376, formula (5): the sign of the third term on the right side $\left(5 \alpha_{0}^{2} \alpha_{2}\right)$, and the sign of the seventh term $\left(\alpha_{1}^{3}\right)$ should be plus.

Page 378, lines 11-12: The expression for $F_{1}$ should agree with the corrected form of formula (5), p. 376; on p. 378 the sign of the last term should be plus.

T. H. Hildebrandt, The Borel Theorem and its generalizations.

Page 453, second footnote: omit the last sentence, beginning "Moore's example, however, is . . ". 